\title{
AN INTEGRATED MICROFLUIDIC INKING CHIP FOR SPM NANOLITHOGRAPHY
}

\author{
Kee Suk Ryu, Xuefeng Wang, Kashan Shaikh, Edgar Goluch, David Bullen, Jun Zou and Chang Liu \\ Department of Electrical and Computer Engineering, University of Illinois at Urbana-Champaign \\ Urbana, IL 61801
}

\begin{abstract}
We report a new architecture of a microfluidic chip for dressing (inking) a high-density array of nanolithography tips in a parallel and multiplexed fashion. The microfluidic chip consists of a matching array of precision patterned thin PDMS films serving as porous inking pads. Inks are supplied from loading reservoirs to the inking pads through embedded microfluidic channels. The thin PDMS membrane allows trapped gas and molecules to permeate through while preventing liquid itself from overflowing or evaporating. The new inking chip provides high density inking, easy loading of inks, and reduced evaporation loss. The fabrication process and results of inking commercial nitride probe and scanning probe contact printing (SP-CP) probes are presented.
\end{abstract}

\section{INTRODUCTION}

Scanning Probe Microscopy (SPM) has been widely used for patterning sub-micron features [1]. The SPM instrument can be used to direct-write chemical and biological molecules with submicron precision for applications such as producing proteomic and gene arrays, functionalizing sensors and circuits, or catalyzing surface chemical reactions. For example, Dip Pen Nanolithography (DPN) [2] utilizes the positioning capability of SPM instruments to deposit chemicals onto substrate with sub-100-nm linewidth. DPN tips are first coated ("inked") with a chemical of interest. When the probe is placed in contact with a writing surface, molecules transfer from the tip to the substrate via meniscus at tip-substrate interface. Recently, a new technique called scanning-probe contact printing (SP-CP) was invented [3]. It utilizes an SPM probe with an integrated tip made of polydimethylsiloxane (PDMS). In the SP-CP process, chemicals are first absorbed into the elastomeric tip. The probe is then moved to a desired location using a commercial SPM. Each contact printing action creates individual dots, analogous to dot-matrix printing. SP-CP process combines the chemical versatility and performance advantage of microcontact printing.

The throughput of SPM-based nanolithography methods can be increased by using arrays of probes [4], arrayed in one- or two dimensions. Certain future applications require inking different tips with different chemicals. However, existing methods for inking DPN or SP-CP probes require exposing tips to vapors or liquids. These inking methods are not suitable for arrayed tips either because of cross-contamination or difficulty of putting different inks to neighboring tips in a dense array (e.g., with tip to tip spacing on the order of $500 \mu \mathrm{m}$ or smaller in one or two axes). Inking using open channels or capillary wells incur rapid ink loss and cross contamination since the ink solution is open to air [5]. In addition, it would require an active control to maintain the level of liquid to interact with multiple tips simultaneously.

A successful method for parallel, multiplicity inking must be practical, scalable (to hundreds of tips per inking session or more), and low cost, while providing high density and low ink loss.

\section{DESIGN AND FABRICATION}

We report a new method of "inking" tips taking advantage of thin $(5 \mu \mathrm{m}$ thick on average) PDMS membrane patches as permeable inking pads. The patches are arranged in array format, matching the density and number of nanolithography tips.

*Travel support has been generously provided by the Transducers Research Foundation and by the DARPA MEMS and DARPA BioFlips programs.

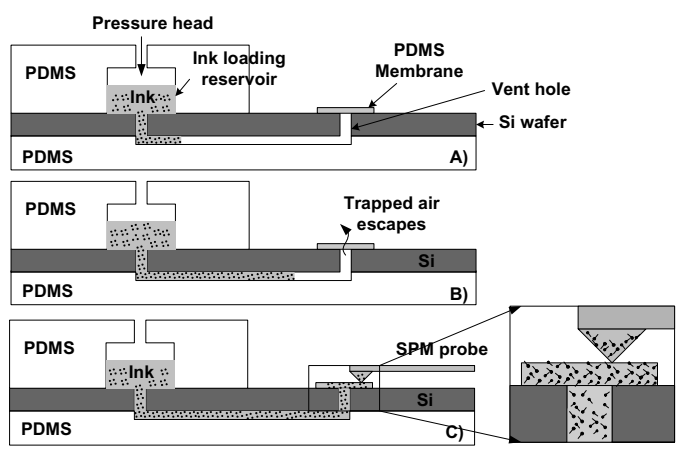

Figure 1. The inking chip with a single inking site shown. PDMS membrane allows gas to escape and the positive pressure head at the reservoir keeps the "ink" at the membrane.

As shown in Figure 1, a thin PDMS membrane (5 $\mu$ m-thick) is patterned in registration with vent holes made in a silicon wafer. Reservoirs and connecting fluidic channels, formed by PDMS molding, are bonded on the top and bottom of the Si wafer, respectively. Once the reservoir is filled with ink (Figure 1(A)), the positive pressure head at the reservoir will move the "ink" towards the inking site (membrane). Trapped air escapes through the thin PDMS membrane (B), allowing inking liquid to contact the bottom of the membrane. The ink, over time, diffused through the membrane to the other side and is picked up by a tip in contact mode. The diffusion of ink molecules through the membrane can be expressed with Fick's first law, i.e., $J=-D(d C / d x)$, where $D$ is the diffusion coefficient of the ink molecule in PDMS, $\mathrm{C}$ is the concentration of the ink in the solution, and $\mathrm{x}$ is the thickness of the PDMS membrane. The diffusion coefficient $\mathrm{D}$ is an experimental parameter that varies with the ink molecules and the PDMS elastomer. Membranes must be thin to allow fast diffusion.

Advantages of this method are following: (1) No active pump or valve required, (2) Cross contamination of adjacent PDMS membranes is minimal, (3) Evaporation of liquid is minimal, (4) high-density inking sites are possible.

We first form throughholes in silicon with DRIE and pattern $\mathrm{Al}$ around the holes (Figure 2). A recently method for making precision PDMS thin film [6] is used to form PDMS patches. The $\mathrm{Al}$ is removed from back. Finally, the top and bottom pieces of PDMS are molded, aligned and assembled. Figure 3 shows the assembled chip with 144 (four groups of $4 \times 9$ arrays) inking sites.

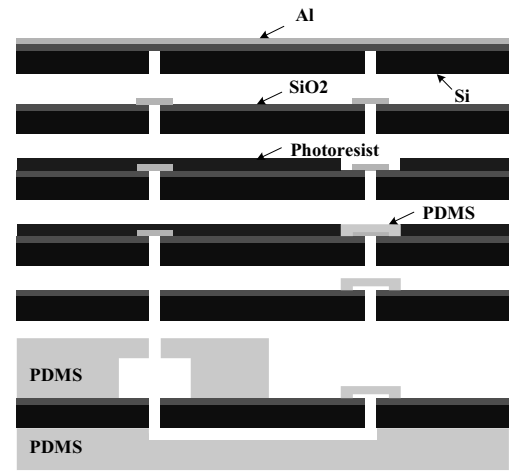

Figure 2. Microfabrication steps including PDMS patterning. 


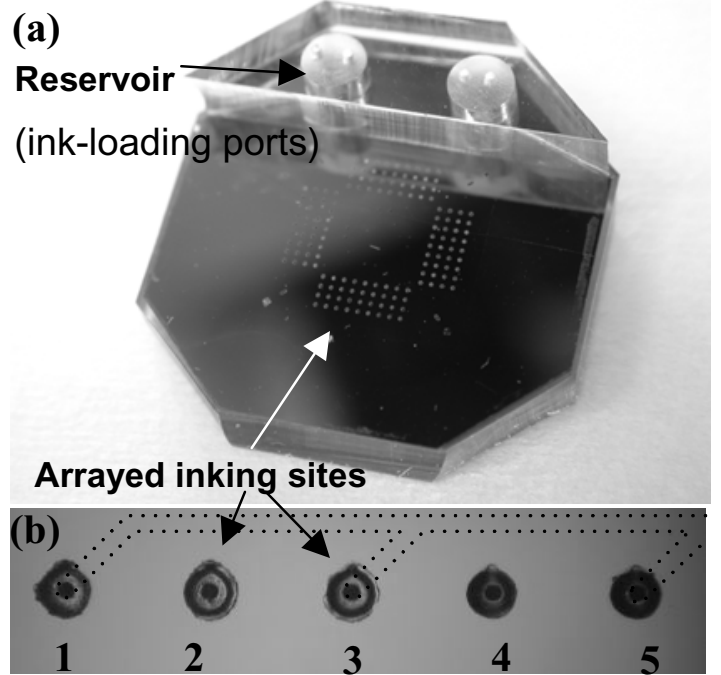

Figure 3. An assembled inking chip with 144 inking sites. (a) Perspective view of chip with two loading ports; (b) Close view of PDMS membranes. The distance to nearby membrane is $500 \mu \mathrm{m}$. Dotted line indicates backside channels.

\section{TEST RESULTS}

Inking of tips by organic molecules has been performed by filling one of the two reservoirs with a $4 \mathrm{mM}$ ethanolic ODT solution. $5 \mu \mathrm{l}$ of the solution was fed into the reservoir. Ink travels to the membrane sites in 2-3 sec. After $6 \mathrm{hr}$ to let the PDMS membrane to be saturated with ODT, SPM tips are made to contact the inking pads to initiate ink transfer from the pad to the tips (Figure 4). Ink loss through vaporization is minimal and can be minimized even more in future. It was found the $5 \mu \mathrm{l}$ of solution completely dries up in about $12 \mathrm{hr}$. With bigger reservoir $(\sim 100 \mu \mathrm{l})$ in the future, the concentration change in the ink can be minimal. Nanolithography tips (including a silicon nitride Microlever tip and a SP-CP tip) were then placed directly on top of the PDMS inking patching but not over the vent hole aperture. After 30 minutes of "inking", the nitride probe and SP-CP probe were used to write on Au surfaces. For comparison, "Inking" by making contact to bulk PDMS soaked for 12-24 $\mathrm{h}$ in a solution at least takes 10 minutes. The inking speed is still limited by diffusion but parallelism and ink multiplexity capabilities are gained.

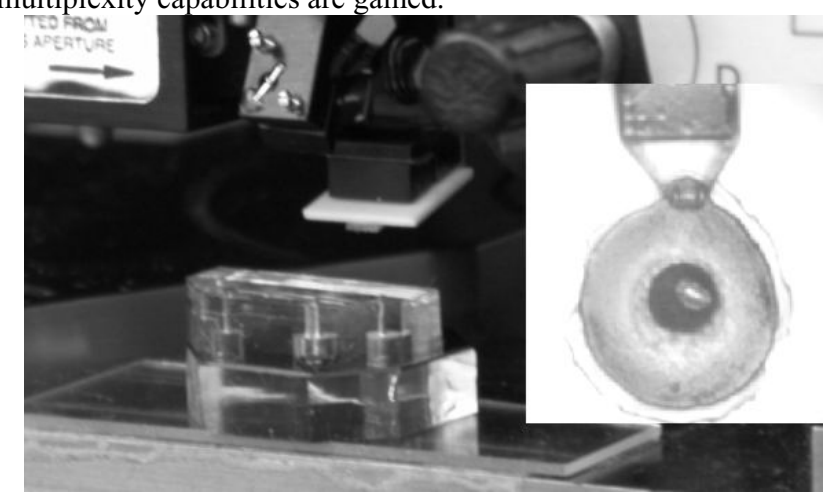

Figure 4. Optical picture of setup in a scanning probe microscope nanolithography station. The inking chip is located below and a SP$C P$ probe is being brought into contact to "ink" the probe. Enclosed optical micrograph shows SP-CP probe being "inked" through PDMS membrane by contact.

Figure 5 (a) shows lines drawn by a commercial nitride probe with the writing speed of $0.1 \mu \mathrm{m} / \mathrm{s}$. Increasing the writing speed results in narrower line widths as shown in Figure 6. Figure 5(b) shows dots drawn by a SP-CP probe. $10 \mathrm{~s}, 30 \mathrm{~s}$, and $1 \mathrm{~min}$ of contact printing resulted in patterns in the sizes of $600 \mathrm{~nm}, 1.3 \mu \mathrm{m}$, and $1.8 \mu \mathrm{m}$.

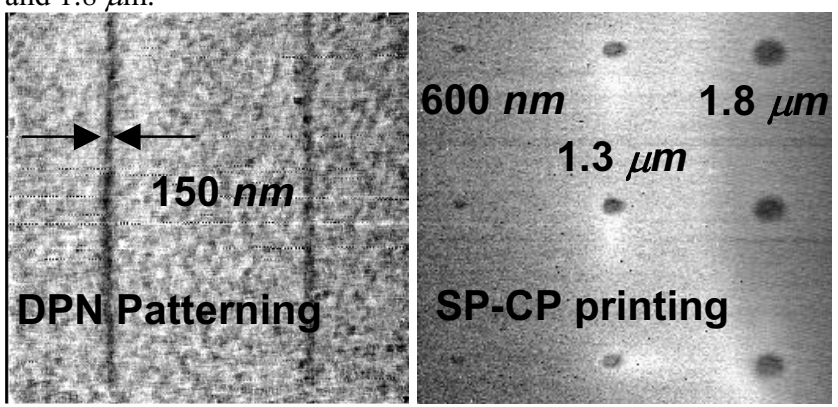

Figure 5. (a) AFM scan image of lines drawn by a nitride DPN tips; (b) ODT dots created by a single SP-CP probe. Large size dots are useful to print coarse features for selected applications.

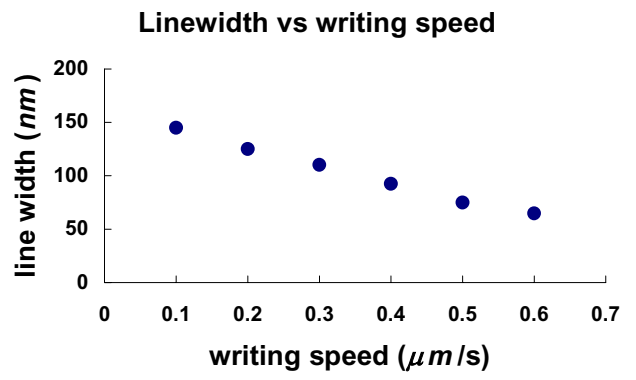

Figure 6. Writing speed and resulting line width plot for commercial nitride probe in DPN writing mode.

Cross contamination was experimentally found to be minimal. We supplied ODT to odd-numbered inkpads (i.e., \#.1, 3, 5 inkpads shown in Figure 3(b)) and making a contact to evennumbered inkpads, which are not being fed by ink. We performed writing after $30 \mathrm{~min}$ of contact but no visible pattern was created by either probe. We concluded that no appreciable cross contamination occurs, $6 \mathrm{~h}$ after filling.

\section{CONCLUSION}

This paper reports a novel method for inking scanning probes for nano lithography with high efficiency. Thin PDMS membranes were used as inkpads and as a membrane which minimize the evaporation of the ink and thus cross-contamination between inking sites. Inking operation has been confirmed. Cross-contamination has been experimentally confirmed to be minimal, at least within 6 hours of filling. The overall inking speed is diffusion limited (even with $5-\mu \mathrm{m}$ thick PDMS membrane) and will be a subject of future improvement.

\section{REFERENCE}

[1] H. T. Soh, K. W. Guarini, and C. F. Quate, Scanning Probe Lithography: Kluwer Academic Publishers, 2001.

[2] R. D. Piner, J. Zhu, F. Xu, S. Hong, and C. A. Mirkin, "'Dip-Pen" Nanolithography," Science, vol. 283, pp. 661-663, 1999.

[3] X. Wang, K. Ryu, D. Bullen, J. Zou, H. Zhang, C. A. Mirkin, and C. Liu, "Scanning Probe Contact Printing," Langmuir, vol. 19, pp. 8951-5, 2003.

[4] D. Bullen, S. -w. Chung, X. Wang, J. Zou, C. A. Mirkin, and C. Liu, "Parallel dippen nanolithography with arrays of individually addressable cantilevers," Applied Physics Letters, vol. 84, pp. 789-791, 2004.

[5] D. Banerjee, J. Fragala, T. Duenas, R. Shile, and B. Rosner, "Planar Capillary Pumped Ink Delivery Apparatus For Dip Pen Nanolithography (DPM)," presented at 7th International Conference on Miniaturized Chemical and Biochemical Analysis Systems, Squaw Valley, CA, 2003.

[6] K. Ryu, X. Wang, K. Shaikh, and C. Liu, "A Method for Precision Patterning of Silicone Elastomer and Its Applications," IEEE/ASME Journal of Microelectromechanical Systems, vol. In press, 2004. 